\title{
The Low-noise Optimisation Method for Gearbox in Consideration of Operating Conditions
}

\author{
Qing Tao ${ }^{1,2}$, Jianxing Zhou, Wenlei Sun, Jinsheng Kang ${ }^{2}$ \\ ${ }^{1}$ School of Mechanic engineering \\ Xinjiang University \\ Urumqi, CHINA 830046 \\ ${ }^{2}$ School of Engineering and Design, \\ Brunel University, \\ Uxbridge, Middlesex, UB8 3PH, UK \\ taoqingx@gmail.com; jianzhou82923@163.com; Sunwenxj@163.com; jinsheng.kang@brunel.ac.uk
}

\begin{abstract}
This paper presents a comprehensive procedure to calculate the steady dynamic response and the noise radiation generated from a stepping-down gearbox. In this process, the dynamic model of the cylindrical gear transmission system is built with the consideration of the time-varying mesh stiffness, gear errors and bearing supporting, while the data of dynamic bearing force is obtained through solving the model. Furthermore, taking the data of bearing force as the excitation, the gearbox vibrations and noise radiation are calculated by numerical simulation, and then the time history of node dynamic response, noise spectrum and resonance frequency range of the gearbox are obtained. Finally, the gearbox panel acoustic contribution at the resonance frequency range is calculated. Based on the conclusions from the gearbox panel acoustic contribution analyses and the mode shapes, two gearbox stiffness improving plans have been studied. By contrastive analysis of gearbox noise radiation, the effectiveness of the improving plans is confirmed. This study has provided useful theoretical guideline to the gearbox design.
\end{abstract}

Keywords: Gear Reducer; Gearbox; Vibration; Noise;

Panel Acoustic Contribution.

\section{INTRODUCTION}

Gear train has been widely used in many industrial fields, with many advantages, i.e. high efficiency, tight structure, and stable speed ratio etc.. When the gear reducer is running, the gearbox vibration is generated, due to the effect of the gear pair dynamic mesh force, which not only affects the stability of the transmission system, but also generates noise. In addition, excessive noise produced by a gear reducer causes operating crew fatigue, stressed, and possible hearing damage. In order to ensure a quiet, smooth, and safe operation of a gear transmission system, it is necessary to understand mechanisms of the dynamic response and the noise radiation of the gear reducer, meanwhile its high reduction ratio is maintained.

With the increasing demand for quieter gear transmission systems, a large amount of work was reported in the literatures on analyzing the vibration and noise of the gearbox. Mohamed et al. built the gearbox vibro-acoustic system by using a three dimensional finiteelement (FE) approach, and the acoustic response of the system was evaluated ${ }^{[1]}$. Velex and Maatar computed the dynamic responses to mesh stiffness variations for gears $^{[2]}$. Their results showed the impact of mesh stiffness variation on dynamic response and tooth loads. Jean et al. developed an experimental and numerical study of dynamic phenomena involving gear impacts with one loose gear inside an automotive gearbox ${ }^{[3]}$. Barthod et al. dealt with the rattle noise, caused by the fluctuation of the engine torque under special conditions, which could cause multiple impacts inside the gearbox ${ }^{[4]}$. Kato et al. simulated the vibration and noise radiation of a singlestage gearbox by combining finite-element (FE) vibration analysis with boundary element noise analysis ${ }^{[5]}$. The results of this analysis were well agreed with the corresponding measured data. Spur and helical gears were tested in the NASA gear-noise rig to compare the noise produced by different gear designs ${ }^{[6]}$. The useful conclusions about the effect of the gear design parameters on gearbox radiated noise were obtained. Choy et al. presented method to predict both the vibration and noise generated by a gear transmission system under normal operation conditions ${ }^{[7]}$, and the application of the method is demonstrated by comparing the numerical and experimental results for the gear noise test rig. Yanyan et al. confirmed that the gear pair is the main excitation of the gear reducer and reduced the gearbox noise through matching the precision grade and stiffness of the gears ${ }^{[8]}$. Kahraman and Blankenship investigated contact ratio effects experimentally using a back-to-back gearbox rig. The dynamic transmission error (DTE) amplitudes of spur gear pairs with varying contact ratios were measured. The measurements were performed for excitation at and around the torsional natural frequency of the gear pair. The gear mesh frequency was used as a form of torsional excitation, with the limitation that excitation is dependent on rotational speed ${ }^{[9]}$. Snežana et al. found that the noise emission of gear units (gearboxes) depends both on the disturbances (gear meshing, bearing operation, etc) and on the insulating capabilities and modal behavior of the housing. Natural vibrations of the housing walls can be prevented or intensified depending on design parameters [10] Tuma review practical techniques and procedures employed to quiet gearboxes and transmission units ${ }^{[11]}$.

In this study, a comprehensive procedure to predict the noise radiation of the gear reducer is presented. In the procedure, a 4 DOFs dynamic model is built, and then taking the bearing force as the excitation, vibrations and noise radiation of the gearbox are studied. According to the results of the panel acoustic contribution analysis on the resonant frequency band of the gearbox and the mode 
shapes, effective methods to reducing vibration and noise are suggested.

\section{ANALYSIS PROCEDURE OF NOISE RADIATION}

Gear errors and fluctuations in mesh stiffness can cause excitation during gear meshing. This excitation propagates from the gear shafts to the bearing, excites the gearbox, and generates gear reducer noise which is radiated from the surface of the gearbox. In order to deal with both gear transmission system dynamic characteristics and gearbox dynamic characteristics, a novel prediction method of gearbox noise radiation is proposed.

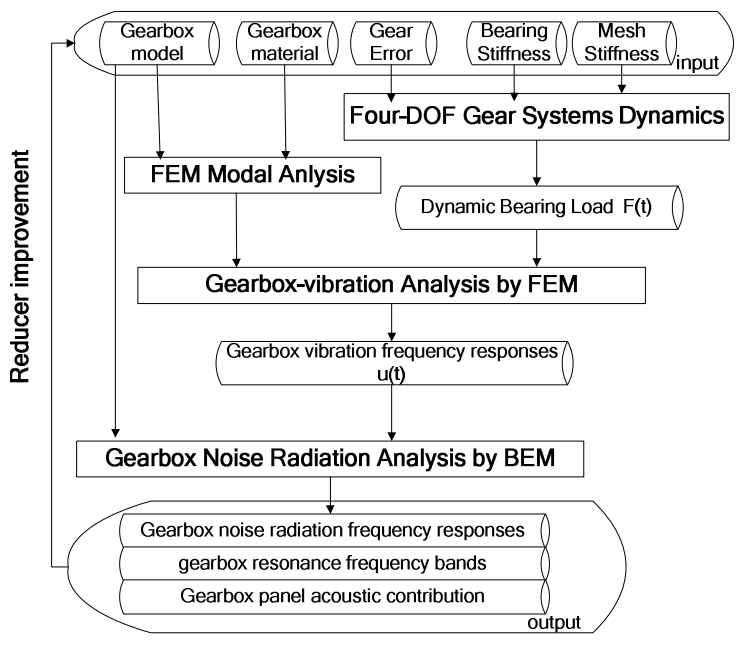

Figure 1. Analysis procedure of gear reducer noise radiation

As illustrated in Fig.1, the developed method consists of three separate steps: dynamic bearing force calculation by solving the gear transmission system dynamic model, gearbox vibration analysis by using finite element method(FEM), and boundary element analysis(BEA) of the sound field. In this method, a commercial software, LMS.Virtual.lab, is used to analyze the sound radiation for the gear reducer. The input data are fundamental performance parameters of the gear reducer, which consist of the gearbox shape, material, gear error, bearing stiffness and so on. The output data are vibrations and noise analysis results, which consist of dynamic responses, frequency spectrum for noise, panel acoustic contribution and so on. The low-noise gearbox is designed according to the conclusion of panel acoustic contribution and gearbox dynamic characteristics.

\section{CALCULATION ON GEARBOX EXCITATION}

In a power transmission gear train, the gear pair assembly remains one of the major noise and vibration sources in the system. The vibrations of the gear transmission system are generated due to the fluctuation of the dynamic meshing force, which is affected by the time-varying mesh stiffness and errors.

\section{A. Gear transmission system excitation}

The gear system parameters are given in Table I. The variation of the mesh stiffness for the gear pair is obtained by using static finite element analysis, in which FEM Contact Algorithm is adopted.

TABLE I. THE GEAR SYSTEM PARAMETERS

\begin{tabular}{|c|c|c|c|c|}
\hline $\begin{array}{c}\text { Power } \\
(\text { Kw })\end{array}$ & $\begin{array}{c}\text { Gear } \\
\text { Ratio }\end{array}$ & $\begin{array}{c}\text { Module } \\
(\mathbf{m m})\end{array}$ & $\begin{array}{c}\text { Pressure } \\
\text { angle }(\mathbf{d e g})\end{array}$ & $\begin{array}{c}\text { Face width }( \\
\mathbf{m m})\end{array}$ \\
\hline 10 & $20 / 80$ & 3.0 & 20 & 60 \\
\hline
\end{tabular}

Since the gear rotation is continuous, the gear meshing stiffness is periodic at the mesh frequency, a complete mesh cycle is divided into several steps, the rotational angle and position of the gears at every step can be calculated according to gear mesh theory. Then the calculation of the mesh stiffness is repeated at every gear engaging position. The time-varying mesh stiffness function is formed by cubic spline interpolation, as shown in Fig. 2(a). As the number of tooth pairs in contact changes, abrupt changes in the gear pair stiffness occur (the mesh of spur gears with two tooth pairs in contact is roughly twice as stiff as when one tooth pair is in contact).
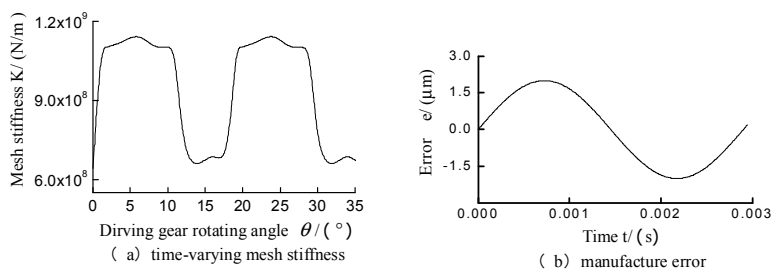

Figure 2. The transmission system excitation

Vibrations of gear pairs are largely affected by the amplitude and phase of deviations of the tooth profile from the true involute one, which is induced by gear manufacturing and installing errors. Meanwhile, with the effect of the gear errors on the instantaneous contact ratio, the collision and impact occur during the gear pair is running ${ }^{[12]}$. As a result, gear errors must be included in the gear transmission system model. Generally, the deviations are assumed to be small enough so that tooth contacts remain on the theoretical line of action. Error function, represent the sum of pitch, profile, pressure angle and run out errors, is supposed as displacement excitations along the tooth profile as a sine wave in the model. The harmonic function is used to simulate the gear error variation which is shown in Fig. 2(b). The error function is written as

$$
e(t)=e_{r} \sin \left(w t / T_{m}+\phi\right)
$$

Where: er is error amplitude, Tm is the mesh cycle, $\mathrm{w}$ is the angular velocity of the driving gear, . is the phase angle. 


\section{B. Gear transmission systems dynamic model}

The proposed dynamic model of the gear pair is shown in Fig. 3, which represents the driving gear (subscript p) meshing with driven gear(subscript $\mathrm{g}$ ). Namely, Kpy and Kgy denote bearing stiffness of the driving gear and driven gear, $\mathrm{Km}$ denotes time-varying mesh stiffness. The angular displacements $\theta p$ and $\theta g$ of the driving gear and the driven gear are in the reversed direction, in the same time, the transverse displacements $\mathrm{Yp}$ and $\mathrm{Yg}$ in the direction of meshing line are also considered.

The angular displacements and the transverse displacements of the gears will affect meshing state of the gear pair, so the displacement is transformed to action line. The displacement on the action line is written as:

$$
\left\{\begin{array}{l}
\bar{y}_{p}=R_{p} \theta_{p}-y_{p} \\
\bar{y}_{g}=y_{g}-R_{g} \theta_{g}
\end{array}\right.
$$

Here: $\mathrm{Rp}$ is the base circle radius of the driving gear. $\mathrm{Rg}$ is the base circle radius of the driven gear.

The mesh force and damping force of the gear pairs are written as:

$$
\begin{aligned}
& F_{K}=k_{m}\left(\bar{y}_{p}+\bar{y}_{g}-e\right)=k_{m}\left(R_{p} \theta_{p}-y_{p}+y_{g}-R_{g} \theta_{g}-e\right) \\
& F_{c}=c_{m}\left(\dot{\bar{y}}_{p}+\dot{\bar{y}}_{g}-e\right)=c_{m}\left(R_{p} \dot{\theta}_{p}-\dot{y}_{p}+\dot{y}_{g}-R_{g} \dot{\theta}_{g}-\dot{e}\right)
\end{aligned}
$$

Where: $\mathrm{e}$ is the gear error. $\mathrm{Rp}$ is the base circle radius of the driving gear. $\mathrm{Rg}$ is the base circle radius of the driven gear. $\mathrm{cm}$ is mesh damping coefficient of gear pair $C_{m}=2 \xi \sqrt{\frac{k_{m}}{1 / m_{p}+1 / m_{g}}}, \mathrm{mp}$ is the mass of the driving gear. $\mathrm{mg}$ is mass driven gear of the driven gear. The damping ratio . is in the range of $0.03 \sim 0.1$.

Therefore, dynamic mesh force $F_{\mathrm{pg}}$ is defined as

$$
F_{p g}=F_{k}+F_{c}
$$

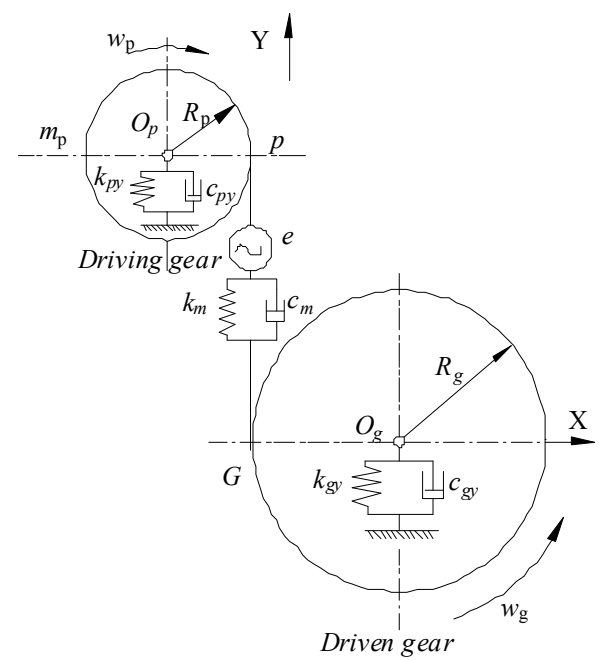

Figure 3. The dynamic model of the system

According to the Newton mechanics law, following differential equations of the gear system are set up, which contains the effects of time-varying mesh stiffness and error excitation. Total number of degree of freedom for the model is 4 .

$$
\left\{\begin{array}{l}
m_{p} \ddot{y}_{p}+c_{p y} \dot{y}_{p}+k_{p y} y_{p}=F_{p g} \\
I_{p} \ddot{\theta}_{p}=T_{p}-F_{p g} R_{p} \\
m_{g} \ddot{y}_{g}+c_{g y} \dot{y}_{g}+k_{g y} y_{g}=-F_{p g} \\
I_{g} \ddot{\theta}_{g}=T_{g}-F_{p g} R_{g}
\end{array}\right.
$$

Here, $\mathrm{Tp}$ is the input torque. $\mathrm{Tg}$ is the load torque. Ip is the rotational inertia of driving gear. Ig is the rotational inertia of driven gear.

The equation of motion is given in the matrix form as

$$
[M]\{\ddot{X}\}+[C]\{\dot{X}\}+K(t)\{X\}=\{P(t)\}
$$

Where: $\mathrm{M}$ is the mass matrix. $\mathrm{C}$ is the damping matrix. $K(t)$ is the stiffness matrix. $X$ is the vector of the displacement. $P(t)$ is the vector of the load.

\section{Dynamic bearing force}

The dynamic bearing force is shown in Fig.4. Dynamic bearing force presents periodic fluctuations and the major components at 4-times, 5-times and 6-times the mesh frequency $(333 \mathrm{~Hz})$.

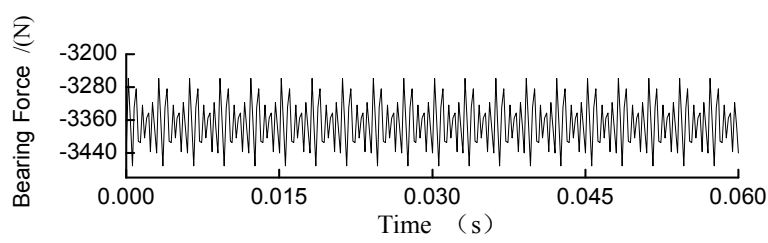

(a) The time history of dynamic pinion bearing force

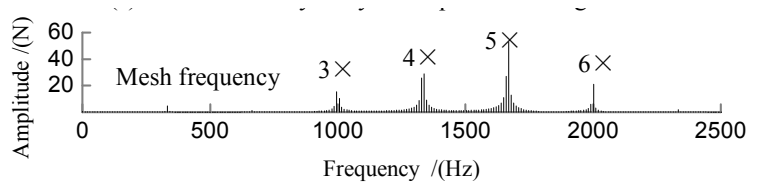

(b) Frequency spectra of dynamic pinion bearing force 


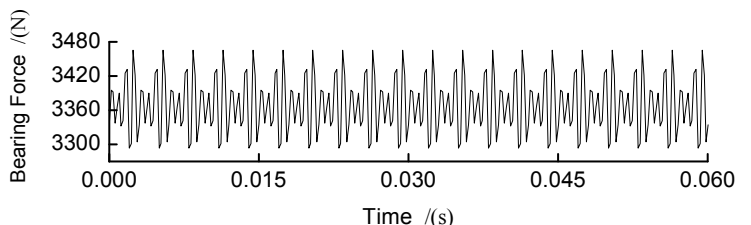

(c) The time history of dynamic wheel bearing force

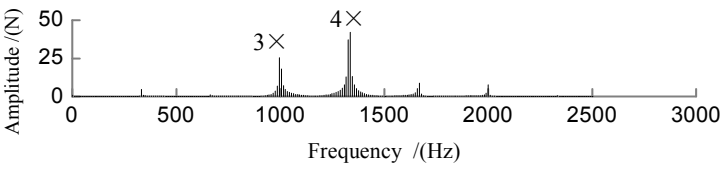

(d) Frequency spectra of dynamic wheel bearing force

Figure 4. The dynamic bearing force

\section{ANALYSIS OF GEARBOX VIBRATION AND NOISE}

\section{RADIATON}

In order to predict the noise of the transmission system during operation, vibration of the gearbox must be accurately computed. The finite element model of the realistic character gearbox is built up by using the commercial software ANSYS and shown in Fig.5. The model consisted of 146238 elements and 38634 nodes. The material of the gearbox is cast steel, whose elastic modulus $\mathrm{E}=207 \mathrm{GPa}$, Poisson ratio $=0.3$, density

$=7800 \mathrm{Kg} / \mathrm{m} 3$. The bolt holes in the bottom of the gearbox are fixed, due to the gearbox connected with the base through the holes. For the convenience of applying dynamic load, a node is created in the center of the bearing bore, then the center node is coupled with the node on the inside surface of the bearing bore and the dynamic load is applied.

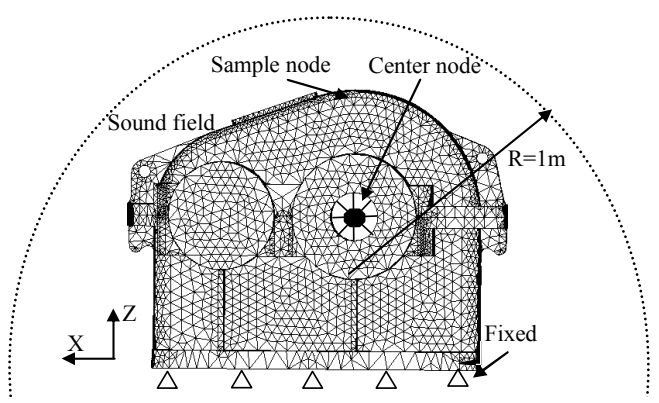

Figure 5. The gear box FE-model

The Lanczos method is used in the modal analysis of the gearbox. Eight modes in the frequency range from 0 to $3000 \mathrm{~Hz}$, shown in fig.6, are chosen to represent the vibration of the gearbox. The vibration of the bottom half gearbox is not as intense as the upper half, because there are bolt constraints and the support of the stiffeners on the bottom of the gearbox.
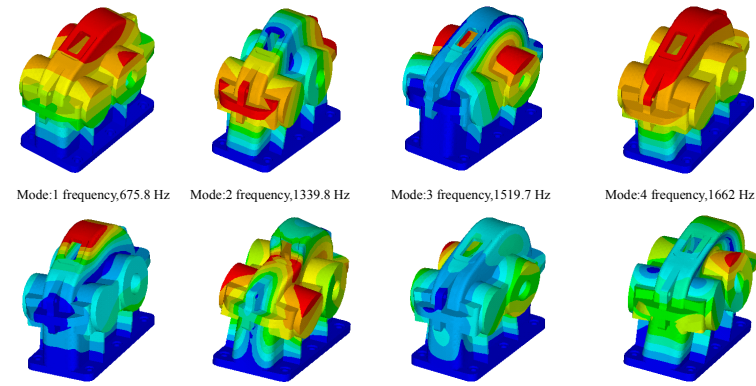

Mode:5 frequency, $2375.3 \mathrm{~Hz}$
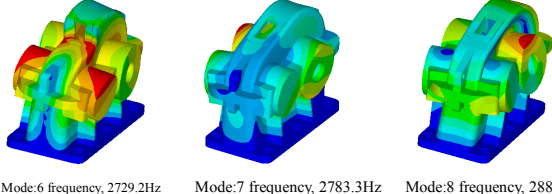

Figure 6. The mode shapes of the gearbox

\section{THE EFFECTS OF ROTATION SPEED ON THE}

\section{VIBRATION AND NOISE}

With change of the rotational speed, not only the gear pair meshing state will change, but also the frequency of the various harmonics will change at the same time. In order to study the effects of the rotation speed, the dynamic bearing force, gearbox vibration and noise radiation are calculated when the rotation speed within the range of $500 \mathrm{r} / \mathrm{min}$ and $3000 \mathrm{r} / \mathrm{min}$.

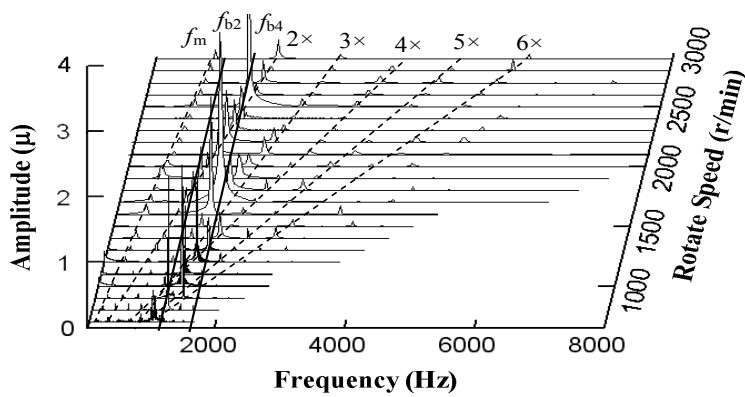

Figure 7. The waterfall of the gearbox dynamic response

Increasing the input speed steadily from $500 \mathrm{r} / \mathrm{min}$ to $3000 \mathrm{r} / \mathrm{min}$, a family of vibration and noise spectrum is obtained. Thus, two waterfall diagrams have been created, as shown in Fig.7 (fm denotes mesh frequency, fb2 and fb4 denote the second and forth nature frequency of the gearbox).

The spectral map illustrates how the various harmonics fall along radial lines and can, thus, be separated from the constant frequency components due to excessive amplification by a structural resonance. The excitation consists of harmonic components whose frequency is a multiple of the corresponding gear's rotational speed, so the major components of response fall along radial lines. Meanwhile, the gearbox produces violent vibration near $1664 \mathrm{~Hz}$ in different rotational speed, since the 2-times the mesh frequency in $2500 \mathrm{r} / \mathrm{min}$, 3-times the mesh frequency in $1600 \mathrm{r} / \mathrm{min}$, 4-times the mesh frequency in $1250 \mathrm{r} / \mathrm{min}$, 5-times the mesh frequency in $1000 \mathrm{r} / \mathrm{min}$ are equal to the fourth natural frequency, the same phenomenon occurs near the second and third natural frequency. So it means that the second, 
third and fourth natural frequency are sensitive to the dynamic bearing force.

The gearbox noise spectral map in $\mathrm{dB}$ is shown in Fig.8. Note that the frequency components of the gearbox noise spectrum are not intense at low speed, as the rotational speed increases, noise radiation was gradually strengthened. The distribution of sound pressure and dynamic response are consistent under various speeds, the resonant frequency band is produced at the range of $670 \mathrm{~Hz}, 1300-1700 \mathrm{~Hz}, 3000-4000 \mathrm{~Hz}$, which are near the gearbox natural frequency. So in order to reduce the gearbox vibration and noise radiation, the vibration at the resonant frequency band should be reduced during the gearbox design stage.

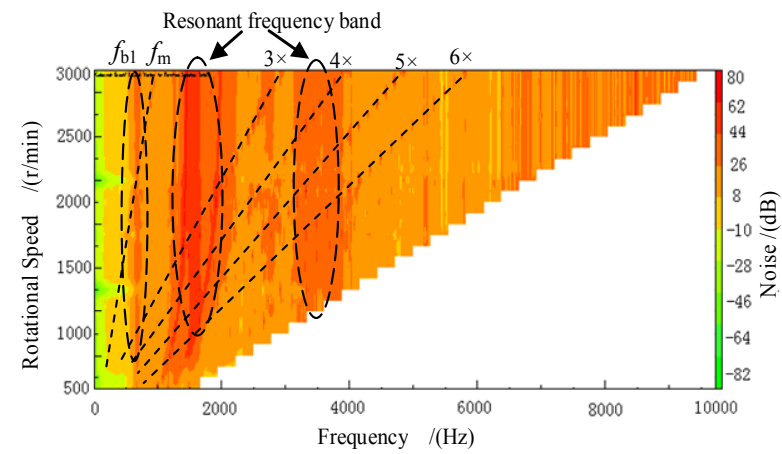

Figure 8. The waterfall figure of the gearbox noise

\section{GEARBOX IMPROVEMENT}

\section{A. Gearbox panel acoustic contribution}

The vibration and noise of the gearbox are sensitive to the shape and structure of its housing. It is necessary to determine the noise contribution of each panel in the resonance region, which provide forceful basis as the gearbox structure is improved.

In order to quantify the noise proportion of each plate to the whole structure, we introduce the concept of panel acoustic contribution coefficient, which is the ratio of the noise pressure produced by vibration of the panels to the overall noise pressure ${ }^{[13]}$.

$$
D_{e}=R_{e} \frac{P_{e} P^{*}}{|P|^{2}}
$$

Where $\mathrm{P}^{*}$ is the conjugate complex number of the sound pressure for the point, $\mathrm{R}$ is its real part. If the phase difference between the panel sound pressure and the overall sound pressure is less than $90 \mathrm{o}$, the overall sound pressure will increase with the raise of the panel vibration velocity, and the contribution coefficient is defined as positive, otherwise, it is negative. The radiating noise can be reduced effectively, if vibration of the panels whose acoustic contributions are positive and values are large can be reduced. Each closed surface of the gearbox is defined as a panel, and the part whose radiation area is too small is neglected, such as the area of corner cutting. The whole gearbox outer surface is divided into 16 panels, as shown in Fig.9.

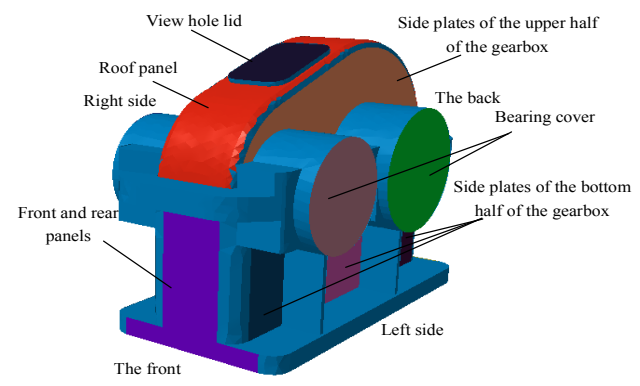

Figure 9. The gear box panel definition

View hole lid(1) ; Roof panel(2) ; Side plates of the upper half of the gearbox $(3,4)$; Bearing cover $(5,6,7,8)$; Side plates of the bottom half

of the gearbox $(9,10,11,12,13,14)$; Front and rear panels $(15,16)$

The panel acoustic contribution coefficient is shown in Fig.10, noted that the contributions of the roof panel and the front and rear panels are greater than other panels when the excitation frequency is $1665 \mathrm{~Hz}$. When the excitation frequency is $667 \mathrm{~Hz}$, the contributions of the roof and side plates of the upper half of the gearbox are bigger. Further analysis indicates that the noise is mainly caused by the panels of the upper half of the gearbox. Reducing of vibration intensity of the panel 2,3,4,15,16 is important to noise control of the gear reducer.
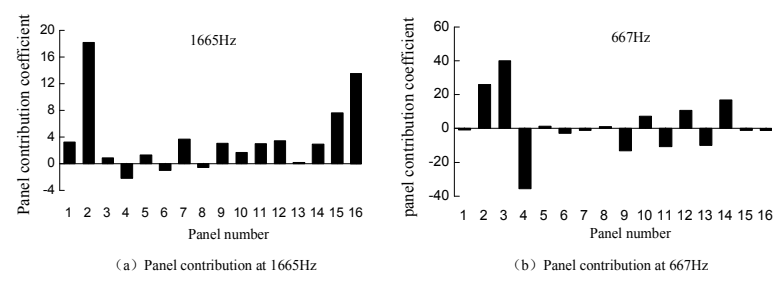

Figure 10. The gear box panel contribution

\section{GEARBOX IMPROVEMENT}

In order to reduce the intensity of vibration of the upper half of the gearbox and make the gearbox natural frequency avoid the 2-times and 5-times the mesh frequency, two low-noise design plans are proposed. The first plan is increase the thickness of the side plates of the bottom half of the gearbox with $4 \mathrm{~mm}$. Another one, the gearbox stiffness was strengthened by using ribs on the side plates, as shown in Fig.11. The natural frequency is shown in Table II.

TABLE II. THE GEAR BOX NATURAL FREQUENCIES ( $\mathrm{Hz})$

\begin{tabular}{|c|c|c|c|}
\hline Mode Steps & Original Model & Plan 1 & Plan 2 \\
\hline $1^{\text {st }}$ & 716.5 & 791.2 & 770.7 \\
\hline $2^{\text {nd }}$ & 1518.8 & 1684.4 & 1631.8 \\
\hline $3^{\text {rd }}$ & 1682.3 & 1847.2 & 1800.1 \\
\hline $4^{\text {th }}$ & 1843.7 & 2138.1 & 2001.4 \\
\hline
\end{tabular}




\begin{tabular}{|c|c|c|c|}
\hline $5^{\text {th }}$ & 2459 & 2887 & 2765.3 \\
\hline $6^{\text {th }}$ & 2881.6 & 3178.4 & 3010.8 \\
\hline $7^{\text {th }}$ & 2887.3 & 3431.6 & 3259.4 \\
\hline $8^{\text {th }}$ & 3043.9 & 3434.3 & 3276.9 \\
\hline
\end{tabular}

The frequency-noise spectra of the gearbox pre and post improvement is shown in Fig.12, where curve a, b, and $\mathrm{c}$ represent the distribution of sound pressure level for the original model and the improved models, respectively. As can be seen, the differences in mesh frequency doubling are considerable. The noise of improved gearbox is reduced obviously when the excitation frequency is below $1700 \mathrm{~Hz}$. The sound pressure level is reduced about $12 \mathrm{~dB}$ at $665 \mathrm{~Hz}$ and about $9 \mathrm{~dB}$ at $1332 \mathrm{~Hz}$ in magnitude.

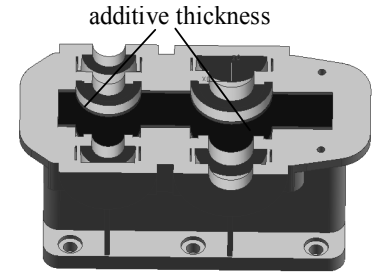

(a) The first low-noise design plan

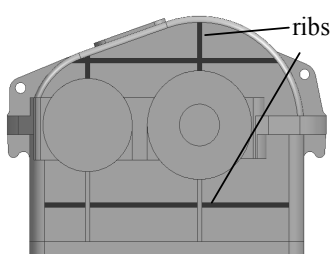

(b) The second low-noise design plan
Figure 11. Improved gearbox

With comparative analysis of the two low-noise design plans, the effect of increasing gearbox thickness and ribs on reducing noise and vibration is almost the same, but the plan 1 will increase more weight and take up more interior room, so it is preferable to provide stiffening ribs on the gearbox.

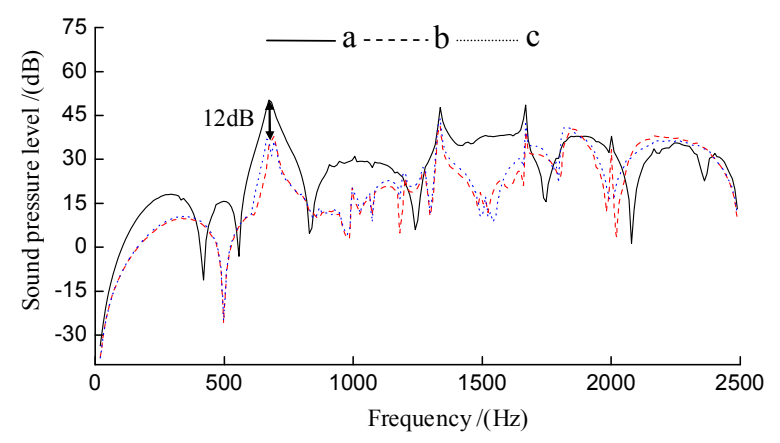

Figure 12. The frequency spectrum for noise

\section{CONCLUSIONS AND SUMMARY}

A procedure for predicting the vibration and noise of gear reducer is developed, in which both gear transmission system dynamic characteristics and gearbox dynamic characteristics are considered. The dynamic bearing force is taken as the excitation, the gearbox vibrations and noise radiation are calculated by using FEM/BEM. The resonant frequency band of the gearbox is obtained. Then the low-noise gearbox was designed base on the result of modal analysis and acoustic panel contribution analysis. It is feasible to reduce the noise radiation of the gearbox through increasing the structural stiffness of the gearbox and reducing the vibration of the panels whose acoustic contribution coefficients are positive and values are large.

\section{REFERENCES}

[1] Mohamed S.A., Slim B.. An acoustic-structural interaction modeling for the evaluation of a gearbox-radiated noise [J]. Mechanical Sciences, 2008,50: 569 577.

[2] Velex P., Maatar M. A mathematical model for analysing the influence of shape deviations and mounting errors on gear dynamic behaviour[J]. Journal of Sound and Vibration 1996,191(5):629 60 .

[3] Jean L.D., Sylvie L.M.. Gear impact and idle gear noise: Experimental study and non-linear dynamic model[J]. Mechanical System and Signal Processing. 2009,23: 2608 $\sim 2628$.

[4] Barthod M., Hayne B.. Experimental study of dynamic and noise produced by a gearing excited by a multi-harmonic excitation[J]. Applied Acoustics.2007,68: 982 1002.

[5] Kato M., Inoue K., Shibata K., et al. Evaluation of sound power radiated by a gearbox[C]. Proc. Inter Gearing'94, 1994. $69 \sim 74$.

[6] Fred B. Oswald, Dnnis P. et al. Influence of gear design parameters on gearbox radiated noise[C], Proc. 1994 International gearing conference, 1994.

[7] Choy F.K., Qian W. et al. Vibration and noise analysis of a gear transmission System[C]. 29th Jiont Propulsion Conference and Exhibit.1993.

[8] Yanyan Z., Zhen G. Analysis and Control of Gearbox Noise[J]. China Mechanical Engineering. 1994,2(5):55 57.

[9] Kahraman A., Blankenship G.W.. Effect of involute contact ratio on spur gear dynamics $[\mathrm{J}]$. Journal of Mechanical Design. Des. 1999, 121:112 118.

[10] Snežana Ćirić Kostić, Milosav Ognjanović. The Noise Structure of Gear Transmission Units and the Role of Gearbox Walls[J]. FME Transactions,2007, 35: 105 112

[11] Tuma J. Gearbox Noise and Vibration Prediction and Control[J]. International Journal of Acoustics and Vibration, 2009,14(2):1 11.

[12] Lin Tengjiao, Jiang Renke. Numerical Simulation of Dynamic Response and Shock Resistance of Marine Gearbox $[\mathrm{J}]$. Journal of Vibration and Shock. 2007,12(26):14 17.

[13] SYSNOISE Rev.5.6, Theoretical Manual. Numerical Integration Technologies, Belgium. 